\section{Chancen und Risiken von Big Data}

\author{
Die Digitalisierung kann unterschiedliche Auswirkungen \\ auf nachhaltige Entwicklungspfade haben. Mit den vielfältigen \\ Chancen von Digitalisierung und Datenrevolution gehen \\ aber auch erhebliche Risiken einher. \\ Von Lucas Wagner und Caroline Hamann
}

D igitalisierung ist ein Megatrend, der immer stärkeren Einzug in alle Bereiche von Wirtschaft und Gesellschaft findet und aus unserer heutigen Welt kaum noch wegzudenken ist. Doch mit dem Internet der Dinge und der vorausgesagten vierten industriellen Revolution stehen noch weitgehendere Veränderungen bevor. Selbst lernende Computer und Roboter ermöglichen individualisierte Dienstleistungen und eine flexible Produktion. Dabei werden Daten zu einer der wichtigsten Ressourcen.

Ebenfalls an Bedeutung gewinnt das Konzept der Nachhaltigkeit. Angesichts globaler Probleme wie des Klimawandels nimmt das Bewusstsein für Verantwortung gegenüber den kommenden Generationen deutlich zu.

Die beiden Konzepte von Digitalisierung und Nachhaltigkeit werden die kommenden Jahre und Jahrzehnte maßgeblich prägen. Vor diesem Hintergrund stellt sich die Frage, wo die Schnittstellen zwischen Digitalisierung und Nachhaltigkeit liegen und inwieweit sich beide Konzepte vertragen. Wo liegen Chancen und Risiken von Digitalisierung und Big Data für eine nachhaltige Entwicklung?

\section{Chancen von Big Data}

Generell eröffnet Big Data viele Möglichkeiten für ressourceneffizientes Wirtschaften sowie für die Individualisierung von Anwendungen, Dienstleistungen und Produkten. Im Zusammenhang mit einer stärkeren Vernetzung bieten sich verschiedene Chancen von Big Data.
Im Bereich der Energieversorgung sind dies beispielsweise intelligente Netze, sogenannte Smart Grids. Virtuelle Kraftwerke und Schwarmenergie bieten weitere Potenziale für eine neue Gestaltung der Energieversorgung.

Eine vernetzte Stadt könnte viele Ressourcen intelligenter verteilen. Dabei spielt auch Mobilität eine wichtige Rolle, denn durch Vernetzung mehrerer Verkehrsmittel kann Mobilität effizienter und individueller gestaltet werden.

\section{Risiken von Big Data}

Mit der Digitalisierung gehen auch Risiken einher. Durch Datenkapitalismus wird der Mensch zur Ware, indem persönliche Daten zwischen Unternehmen gehandelt werden. Gleichzeitig ist auch die Eigentumsfrage der Daten nicht eindeutig geklärt. Die Macht der Oligopolisten im Internet, also der Marktteilnehmer, die nur in geringem Wettbewerb stehen, steigt also weiter.

Ferner bestehen Risiken durch den disruptiven Charakter vieler digitaler Geschäftsmodelle, wie es am Beispiel Uber oder AirBnB sehr deutlich wird. Meist sind es Kostenexternalisierungen, die diesen Firmen zum Erfolg verhelfen. Durch die vierte industrielle Revolution werden allerdings nicht nur einzelne Geschäftsmodelle verdrängt. Auch Berufe oder sogar ganze Wirtschaftszweige werden durch Computer ersetzbar. Was ist am Ende der Mensch als Arbeitskraft noch wert, wenn er gegen Roboter und Computer konkurriert?
Im Spannungsfeld von Digitalisierung und nachhaltiger Entwicklung wird auch der Kontrollverlust des Menschen zum Thema. Bereits heute wird dieser an den Börsen erkennbar. Hier bestimmen Algorithmen über Milliarden und Computer handeln in Pikosekunden.

Darüber hinaus ist auch der Einfluss von sozialen Medien wie Facebook und Twitter auf die Gesellschaft nicht zu unterschätzen. Insbesondere junge Menschen verbringen viel Zeit vor ihren Endgeräten und flüchten in virtuelle Illusionswelten. Reale zwischenmenschliche Kontakte leiden dadurch oftmals.

\section{Gesellschaftlicher Mehrwert der Digitalisierung}

Zusammengefasst bietet die Digitalisierung nie dagewesene Möglichkeiten, um neue Erkenntnisse zu gewinnen.

Die Datenrevolution schafft daher ohne Zweifel Lösungen für eine Vielzahl an Herausforderungen. Fraglich jedoch ist, ob es Antworten und Lösungen zu den drängenden Problemen unserer Zeit sind. Generiert Datenkapitalismus und Digitalisierung letztendlich gesellschaftlichen Mehrwert jenseits des Wachstums? Wer profitiert von diesem Wachstum?

Diese und weitere Fragen werden im November 2015 auf der größten Konferenz im deutschsprachigen Raum zu diesem Thema mit Vertretern aus Wirtschaft, Wissenschaft, Politik und Religion diskutiert. [1]

\section{Anmerkungen}

[1] „Digitalization meets Sustainability“ lautet das Thema der größten studentischen Konferenz zum Thema Nachhaltigkeit im deutschsprachigen Raum, dem WFI - Dialogue on Sustainability, die vom 20. bis 22. November 2015 in Ingolstadt stattfindet.

AUTOR + KONTAKT

Caroline Hamann ist Mitglied des Vorstandes von DenkNachhaltig e. V. Lucas Wagner ist Mitarbeiter in der Geschäftsstelle von DenkNachhaltig e. V. WFI Ingolstadt School of Management, DenkNachhaltig e. V., Auf der Schanz 49, 85049 Ingolstadt. Website: www.denknachhaltig.de 